\title{
Month of birth and schizophrenia in Taiwan: effect of gender, family history and age at onset
}

\author{
Wei J. Chen ${ }^{\mathrm{a}, *}$, Ling-Ling Yeh ${ }^{\mathrm{b}}$, Ching-Jui Chang ${ }^{\mathrm{b}}$, Li-Chi Lin ${ }^{\mathrm{b}}$, Hsien Rin ${ }^{\mathrm{b}}$, \\ Hai-Gwo Hwu ${ }^{\text {b }}$ \\ a Institute of Epidemiology, College of Public Health, National Taiwan University, No. 1, Sec. 1, Jen-Ai Rd., Taipei, \\ Taiwan \\ ${ }^{b}$ Department of Psychiatry, College of Medicine, National Taiwan University, Taipei, Taiwan
}

Received 1 February 1995; accepted 20 July 1995

\begin{abstract}
Schizophrenic patients have been shown to have a moderate excess of winter births in the areas where seasonal variations in weather are large. In this report, we examined the seasonality of schizophrenic births in Taiwan, which has a subtropical climate. Using nationwide hospitalization data ( 2429 male and 1320 female schizophrenic patients), we applied the life table method to compare the risk of schizophrenia among 12 cohorts of month-of-birth for males and females, respectively. Differences among the risks of the 12 cohorts were tested using the logrank test. The samples were further stratified by family history and age at onset. There was a significant association between the risk of being admitted as a schizophrenic and month of birth for both males and females. The cohorts born in November and January had the highest risks. After stratification, the association was significant only for non-familial, male, and early onset schizophrenic patients. The results indicate that seasonally varying factors might increase the risk of schizophrenia, especially in those without a family history of the disease. Men are more vulnerable to such factors than women, and the schizophrenics resulting from such insults tend to be early onset.
\end{abstract}

Keywords: Age at onset; Family history; Gender; Month of birth; (Schizophrenia)

\section{Introduction}

Schizophrenic patients have been shown to have an excess of births in the winter or early spring as compared to controls (Bradbury and Miller, 1985; Boyd et al., 1986; Hare, 1988; O'Callaghan et al., 1991a; Torrey et al., 1993; Pallast et al., 1994). Although earlier studies of season of birth for

\footnotetext{
* Corresponding author. Tel.: +886 (2) 397-0800, ext. 8360; Fax: $+886(2)$ 356-0840.
}

schizophrenia suffered from inadequacies in case definition and selection of controls, at least 13 studies using sound methodology have been published (Bradbury and Miller, 1985). One major improvement in these studies was that they chose the number of births from the general population in the same years of schizophrenic births as controls.

Even so, studies ascertaining cases by calendar year might still have an age-prevalence effect artifact (Lewis and Griffin, 1981). That is, individuals born in January are 11 months older than individ- 
uals born in December of the same calendar year and hence the former are over-represented among prevalent cases. Some researchers have responded to this critique and applied methods that correct or avoid the age-prevalence artifact (Watson et al., 1982; Pulver et al., 1983; Shur and Hare, 1983). Their results showed that the seasonality of schizophrenia remained after such adjustment. Many researchers have also pointed out that several findings in the seasonality of schizophrenia are inconsistent with the prediction of age-prevalence effect, such as an excess of schizophrenic births in December or the last quarter of a year in the northern hemisphere, an excess of schizophrenic births in the middle of the year in the southern hemisphere, and lack of season-of-birth effect for other minor psychiatric disorders (Bradbury and Miller, 1985; Boyd et al., 1986; Hare, 1988; Dalen, 1990; Pulver et al., 1990; Torrey and Bowler, 1990; Watson, 1990). Thus, the seasonality of schizophrenic births is not an artifact due to the ageprevalence effect.

Traditionally, the seasonality of schizophrenic birth was evaluated via a $\chi^{2}$-est comparing the observed number of births of schizophrenics with the expected births computed from the general population. To correct for age-prevalence effect, either the risk period was incorporated in the computation of expected births or a different definition of a calendar year was used (Lewis and Griffin, 1981). Pulver et al. (1983) applied the life table method to calculate the cumulative risk of schizophrenia for cohorts of different birthmonths. This method avoids the age prevalence effect completely, as cumulative risks for the same length of time were compared. Furthermore, the risks calculated by this approach could be compared to known morbidity risk figures in the population, to assess the representativeness of the sample used.

The nature of the observed seasonality of schizophrenic births is still unclear. It is commonly hypothesized that some seasonally varying factors, such as temperature, nutritional deficiencies, infectious agents, and obstetric complications are responsible for an individual's subsequent development of schizophrenia (Bradbury and Miller, 1985; Boyd et al., 1986; Hare, 1988; Torrey et al., 1993).
Regardless of which explanation is more likely, presumably the larger seasonal variations of weather, the larger the magnitude of the seasonality of schizophrenic births, and vice versa. So far, seasonality of schizophrenic births has been found mainly in areas where seasonal variations in weather are large, such as northern America (USA, Canada), northern Europe (England and Wales, Sweden, Denmark), Japan and Australia. Torrey and Torrey (1979) reported that the seasonality of schizophrenia diminished as the area moved from the northern states to the southern states in the US. However, one study in the Philippines (Parker and Balza, 1977) found seasonal effects for schizophrenia despite there being no true winter in that equatorial area.

The effect of seasonality on schizophrenia, nevertheless, is minor. Pulver et al. (1983) showed that the risk of schizophrenia for those born during winter was only $5-15 \%$ higher than that for the rest of the year. Thus the season of birth may be a risk factor only for a subgroup of schizophrenic patients. Many researchers have examined the seasonality effect on subsamples divided on the basis of covariates such as gender, family history, chronicity, marital status and severity (Bradbury and Miller, 1985). However, the results were not conclusive.

In this study, we applied the life table method to analyze data from Taiwan, which is located in a subtropical area, to determine whether there is an increase in the risk of schizophrenia for those born in the winter or early spring. In addition, we stratified schizophrenic patients by gender, family history, and age at onset into subsamples to examine the effect of these covariates on the seasonality of schizophrenic births.

\section{Methods}

\subsection{Study population}

The schizophrenic patients included in this study were derived from the Taiwan Inpatient Epidemiological Project (TIEP). The background and procedure of TIEP have been described in detail elsewhere (Hwu et al., 1995). In brief, any 
Taiwanese person who received inpatient treatment for mental illness at any time between October 1, 1987 and September 30, 1988 were enrolled in TIEP. In total, there were 17,508 psychiatric inpatients during the 1-year period of TIEP. Out of 58 psychiatric hospitals in Taiwan, only 9 small hospitals did not participate in this project because of closure of inpatient service or refusal. The number of beds provided by these 9 hospitals was only $2.2 \%$ of the total number of psychiatric beds in Taiwan in 1987. The number of enrolled patients accounted for about $88 \%$ of all psychiatric inpatients reported to the Department of Health. All the 49 hospitals participating in TIEP were requested to complete a data sheet for each patient with information regarding his or her demographic status and clinical features (including diagnosis, treatment history, age at onset, family history, etc.).

Diagnoses of patients were provided by hospitals participating in TIEP and were classified according to ICD-9 (World Health Organization, 1978). For schizophrenic patients, their age at onset was defined as the first time that their psychotic symptoms became prominent and began to impair their daily activities. The median age at onset for our sample was 21 years, which was similar to the mean ages at first psychotic episode (20-24 years) for schizophrenic patients reported by Pulver et al. (1992).

The psychiatric status of the first-degree relatives of patients was assessed via either the patients themselves or informants (usually care-givers). If one or more first-degree relatives of a schizophrenic patient was reported to have psychotic or affective symptoms that led to severe impairment of daily activities or admission for inpatient treatment, the family history was considered positive. Otherwise, the family history was considered negative.

The inter-rater reliability of the recorded data was assessed in a smaller sample of 70 patients (Hwu et al., 1995). For demographic data, $\kappa$-values (for categorical variables) or intraclass coefficient reliabilities (for ordinal or continuous variables) were $\geq 0.70$ for $96 \%$ of the questions on the data sheet. As to clinical data, the intraclass coefficient reliabilities of 12 ordinal questions were $\geq 0.90$, while $\kappa$-values for the $96 \%$ of the remaining categorical questions were $\geq 0.70$. For this study in particular, the intraclass coefficient reliability for age at onset was 0.89 and $\kappa$ for family history of schizophrenia was 0.80 . In general, the reliability of the data collected is satisfactory.

Among the patients who had been diagnosed, 11,971 patients $(70.0 \%)$ were diagnosed as having schizophrenia. As there were no vital statistics for numbers of births in each month before 1952 in Taiwan, patients born before that year were not included in the analysis. For those born after 1966, their ages at ascertainment (1987) would be less than 20 years and so they were omitted from the analysis as well. Patients with data missing for age at onset or family history were also excluded. In total, there were 3749 schizophrenic patients ( 2429 males and 1320 females) used in the analysis.

The population at risk from which the schizophrenic patients came was comprised of individuals born between 1952 and 1966 in Taiwan. These data were obtained from vital statistics published by the Department of Health, Taiwan Provincial Government.

\subsection{Data analysis}

The schizophrenic patients and the population at risk were divided into 12 month-of-birth-specific 'pooled cohorts', separately for males and females (Table 1). Within each cohort, the risk of being admitted as a schizophrenic at different age intervals was estimated by the technique of actuarial life table (Lee, 1980).

In the construction of a life table, we divided the age into 7 intervals $(<1,1-4,5-9,10-14$, 15-19, 20-24 and 25-29 years). The reason we did not calculate risk up to an age interval beyond 25-29 years was that most of the study population would have been censored at that interval. For each month-of-birth cohort, three kinds of information needed to complete the calculations are listed below.

(1) Initial number at the beginning. This was obtained by pooling the number of births between 1952 and 1966. However, since the inpatients in our sample comprised only $88 \%$ of all inpatients, we adjusted this number by multiplying by 0.88 . 


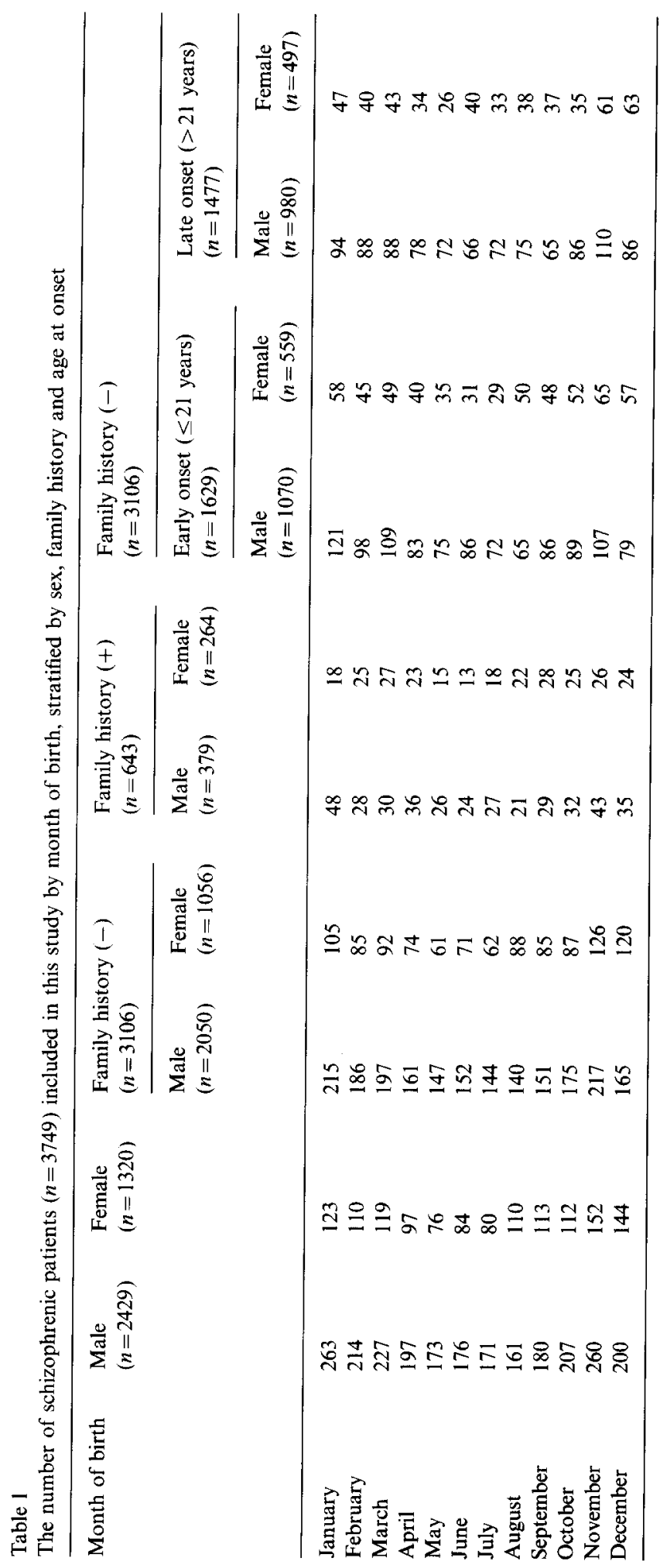


(2) Number censored. Any subject who had not yet been admitted as a schizophrenic or had died before the end of an age interval was censored. This was estimated by the age-specific mortality rate obtained from life tables published by the Committee on Construction of Life Tables, Taiwan Provincial Government. We used mean age-specific mortality averaged over 15 years (1952-1966) for the calculation. Thus, not only neonatal deaths, but also mortality at other ages were used to adjust for the initial live birth frequencies. Because we pooled subjects of different birth years together, a whole sub-cohort born in an earlier year would be censored after a certain number of years.

(3) Number of events. The number of schizophrenic patients whose age at onset occurred within an age interval was counted as the number of events.

The cumulative probability of being admitted as a schizophrenic up to a particular age interval was calculated as one minus the survival probability of being free from the event up to that age interval. The standard error of the cumulative probability was calculated according to the Greenwood formula (Lee, 1980). The differences in life table risk among each of the 12 month-of-birth cohorts were examined by the logrank test (Peto et al., 1977).

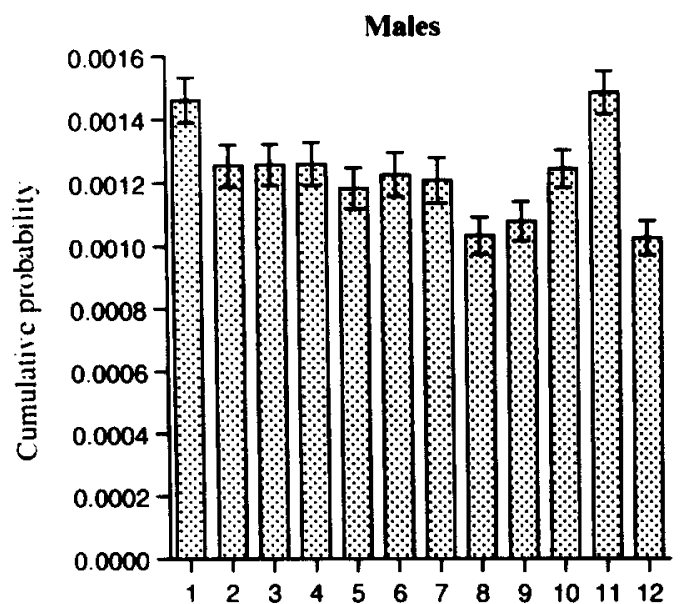

The schizophrenic samples were then divided into subsamples of those without a family history and those with a family history, separately for males and females (Table 1). Procedures similar to the preceding ones were performed to test the seasonality of schizophrenic births in subsamples. For those without a family history, we then subdivided the sample into those with early onset and those with late onset. Since there was no consensus regarding the definition of early onset, we arbitrarily chose the median age at onset ( 21 years) as the cut-off point to make the two subsamples equal in statistical power in detecting the seasonality of schizophrenic births. Thus we subdivided the sample into those with age at onset of 21 years and younger (early onset) and those with age at onset over 21 years (late onset). For those with a family history, we did not subdivide them further because the number of patients was too small.

\section{Results}

The cumulative probability of being admitted as a schizophrenic between the ages of 25 and 29 years, with corresponding standard errors for males and females, born between 1952 and 1966

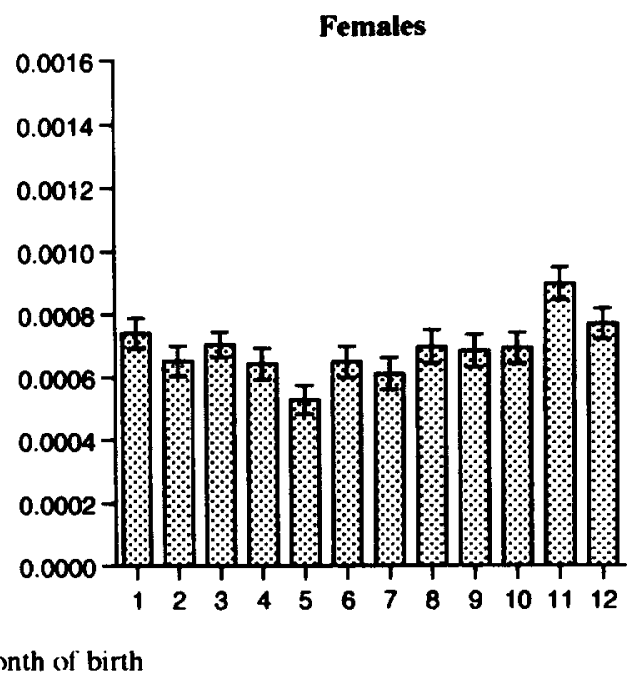

Fig. 1. The cumulative probability of being admitted as schizophrenic between the ages of 25 and 29 years for Taiwan residents born between 1952 and 1966 by month of birth and sex. The standard error of each estimate is indicated by the bar. (The logrank test of homogeneity for risks of 12 cohorts: $\chi^{2}{ }_{11}=36.0, p<0.001$ for males and $\chi^{2}{ }_{11}=19.6, p=0.05$ for females.) 
by month of birth are displayed in Fig. 1. The logrank test of homogeneity for the risks of schizophrenia among 12 month-specific cohorts was rejected statistically for both males and females. However, the patterns of the risks of schizophrenia were different between males and females. For males, the risks of being admitted as a schizophrenic were highest for cohorts born in January and November and lowest in December and August. As for females, while the risk was the highest among the cohorts born in November, followed by December and January, the May and July cohorts had the lowest risks.

By temperature alone, 12 months could be grouped into 4 seasons (December, January and February for winter; March, April and May for spring; June, July and August for summer; and September, October and November for autumn). However, there were no differences in the risk of schizophrenia among cohorts of the 4 seasons for either males or females (data not shown).

When the sample of schizophrenic patients was divided into subsamples of those with and without a family history, the association between the risk of schizophrenia and month of birth was significant only for those without a family history. For those without a family history (Fig. 2), the logrank test of homogeneity for risks of 12 cohorts was rejected statistically both for males and females. For those with a family history (Fig. 3), the logrank tests were not significant for either males or females.

The sample of schizophrenic without a family history was further subdivided into early and late onset. For the early onset subsample, the association between the risk of schizophrenia and month of birth was significant only for males (Fig. 4). However, the pattern of risk among male early onset subsample was scattered. The risk of schizophrenia for cohorts of January, March, November, June and February were among the highest, while those for December and August were among the lowest. There was no association between the risk of schizophrenia and month of birth for either male $\left(\chi_{11}^{2}=13.1, p>0.25\right)$ or female $\left(\chi_{11}^{2}=17.0\right.$, $p>0.10$ ) late onset subsamples.

\section{FAMILY HISTORY: NEGATIVE}
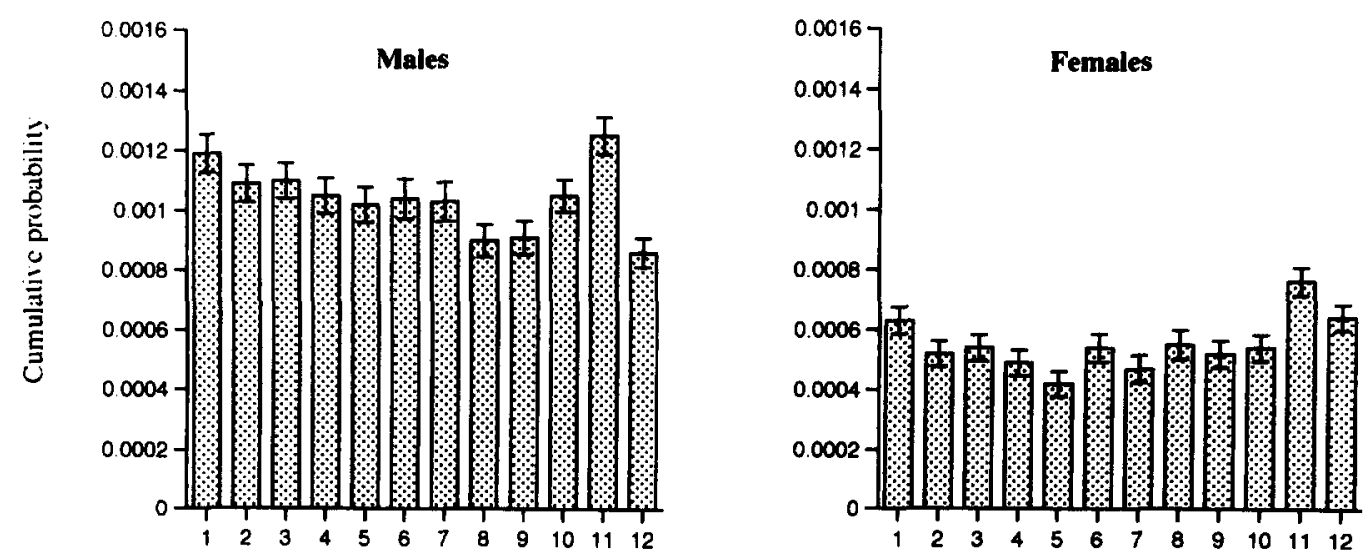

Cohorts by month of birth

Fig. 2. The cumulative probability of being admitted as schizophrenic with no family history between the ages of 25 and 29 years for Taiwan residents born between 1952 and 1966 by month of birth and sex. The standard error of each estimate is indicated by the bar. (The logrank test of homogeneity for risks of 12 cohorts: $\chi_{11}^{2}=27.8, p<0.005$ for males and $\chi^{2}{ }_{11}=21.8, p=0.05$ for females.) 


\section{FAMILY HISTORY: POSITIVE}
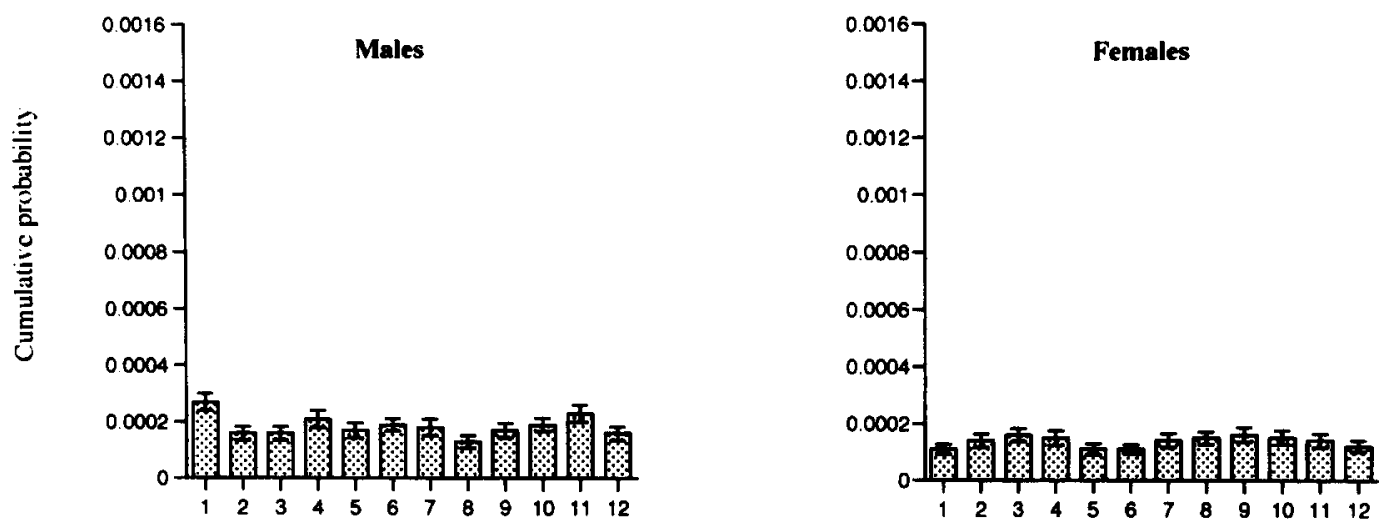

Cohorts by month of birth

Fig. 3. The cumulative probability of being admitted as schizophrenic with a family history between the ages of 25 and 29 years for Taiwan residents born between 1952 and 1966 by month of birth and sex. The standard error of each estimate is indicated by the bar. (The logrank test of homogeneity for risks of 12 cohorts: $\chi^{2}{ }_{11}=15.1, p>0.10$ for males and $\chi^{2}{ }_{11}=7.3, p>0.75$ for females.)

\section{FAMILY HISTORY: NEGATIVE AGE AT ONSET $\leq 21$ YEARS}
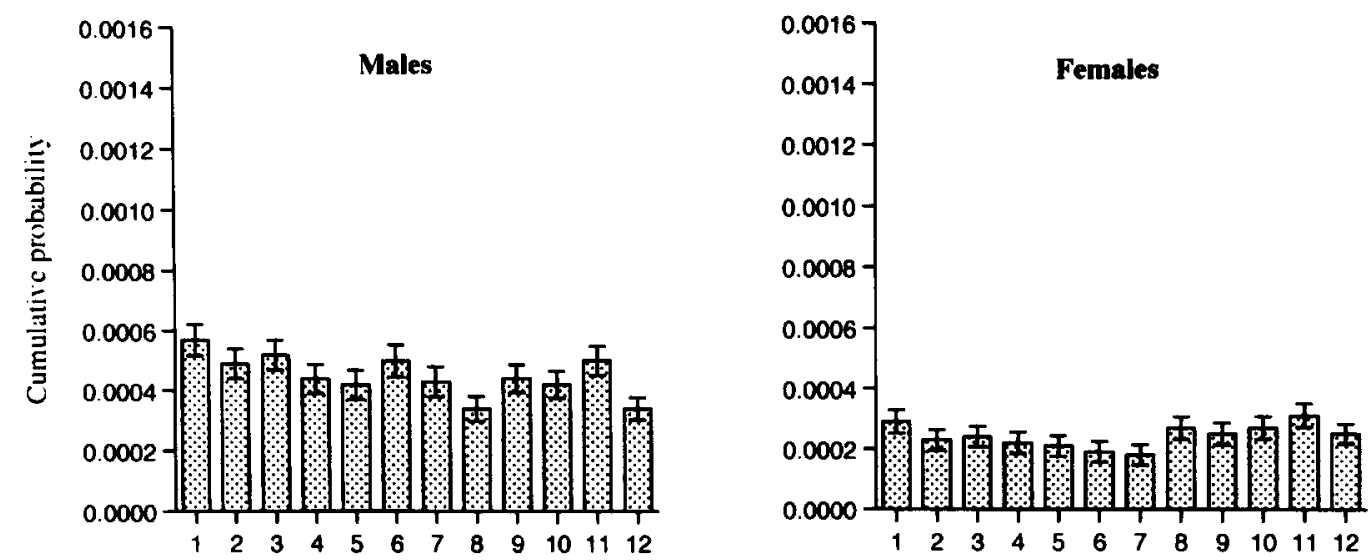

Cohorts by month of birth

Fig. 4. The cumulative probability of being admitted as schizophrenic with no family history and an early onset less than or equal to age 21 years for Taiwan residents born between 1952 and 1966 by month of birth and sex. The standard error of each estimate is indicated by the bar. (The logrank test of homogeneity for risks of 12 cohorts: $\chi^{2}{ }_{11}=25.2, p<0.01$ for males and $\chi^{2}{ }_{11}=11.7, p>0.25$ for females). 


\section{Discussion}

Despite the fact that seasonal variations of weather in Taiwan are very mild, our results showed that there was an association between the risi of schizophrenia and month of birth. Previously, Parker and Balza (1977) also reported that there were excess schizophrenic births in the cold season (i.e., December, January and February) in the Philippines, which is located in an equatorial area and has no real winter. Meanwhile, there was no difference in the number of births if the patients were subgrouped as those born in the wet season versus those born in the dry season in the Philippines.

However, the pattern of the risk of schizophrenia in our data was not compatible with the prediction solely based on seasonal variations in temperature (Fig. 1). For example, the 3 months of the lowest temperature in Taiwan are December, January and February, while those of the highest are June, July and August. In this report, the risk of schizophrenia for the cohort of November was the highest for males, while that for the cohort of December was the lowest. If we grouped 12 months into 4 quarters based on temperature alone, there were no longer significant differences in the risk of schizophrenia among them. Torrey and Torrey (1979) also found that temperature alone could not explain the progressive shift of the greatest excess in schizophrenic birth from February to April and May over a 3-decade period in the US.

There were some limitations of our data that should be mentioned. First, we included admitted schizophrenic patients only. Second, the ascertainment period was 1 year long. Cases not admitted during this year would not be included. Both might lead to underestimation of risk. There have been 3 community surveys of mental illnesses in Taiwan, i.e., the survey in 1946-1948 (Lin, 1953), the survey in 1961-1963 (Lin et al., 1989) and the survey in 1982-1986 (Hwu et al., 1989). According to the results of these 3 surveys, the estimated lifetime prevalences of schizophrenia by temporal order were $0.002,0.0012$ and $0.0019-0.0037$ for males and $0.0012,0.0016$ and $0.0007-0.0032$ for females. When these figures were compared to the cumulative risks derived from our data (Fig. 1), we found that there was an underestimation in the cumulative risks, but not by much. However, the underestimation did not occur preferentially in any particular cohort of month-of-birth. Thus, our comparisons between cohorts born in different months are still valid.

The risk of being admitted as a schizophrenic for males was almost twice that for females in our data. In the study of Pulver et al. (1983), in which they compared the risk of being diagnosed as schizophrenic, the risk among males was approximately equal to that among females. Possible reasons for this difference are: (1) the symptoms of female schizophrenic patients tended to be less severe and of shorter duration than males (Goldstein and Link, 1988); and (2) female patients were more tolerated at home than males. These factors might lead to a lower risk for being admitted among females. However, this would not affect the validity of comparing females themselves among 12 month-of-birth cohorts.

Although there appeared to be an association between the risk of schizophrenia and month of birth for both males and females in our data, the trend in females was less prominent than that in males. This differs from previous reports. Nine of 10 previous studies reviewed by Bradbury and Miller (1985) showed that there were no gender differences, or only a slight winter excess for female patients in the seasonality effect of schizophrenia. One study reported a significantly greater seasonality effect for schizophrenia among women in the US (Pulver et al., 1981). Recently, Pulver et al. (1992) using morbidity risk analysis reported a greater seasonality effect for women.

Our data showed that the seasonality of schizophrenia only exists in non-familial schizophrenia and not in familial schizophrenia in both males and females. However, there were some potential pitfalls in our definition of familial vs. non-familial forms of schizophrenia. As pointed out by Kendler (1988), relying on family history in defining familial vs. non-familial forms of schizophrenia has a limited discriminating power. The validity of the family history reported in our data might vary across hospitals that participated in TIEP. Nevertheless, the proportion of patients with any first-degree relatives affected with major psychiat- 
ric disorders in our data (17\%) was similar to the $18 \%$ reported by Baron and Gruen (1988), and the $19 \%$ reported by O'Callaghan et al. (1991a).

The results from 11 previous studies that examined the relationship between family history and the seasonality effect of schizophrenia are contradictory. Most of them compared the distribution of birth dates of familial schizophrenic patients with those of non-familial patients. Six of these studies found that non-familial patients were more likely to be born in winter than familial ones (Kinney and Jacobsen, 1978; Shur, 1982; McNeil, 1987; Zipursky and Schulz, 1987; Sacchetti et al., 1989; O'Callaghan et al., 1991a), two reported that familial patients are more likely to be born in winter (Lo, 1985; Owen et al., 1989), and two found no difference between the two subgroups (Baron and Gruen, 1988; Kim et al., 1994). In addition, there were studies that examined the risk of schizophrenia among the relatives of schizophrenic patients born in different months. Both Baron and Gruen (1988) and Pulver et al. (1992) found that the relatives of schizophrenic probands born in winter had the highest risk.

Another interesting feature of our results was the interaction of gender and age at onset with the seasonality effect of schizophrenia among patients without a family history (Fig. 4). Of the 4 subsamples classified by age at onset (early vs. late) and sex, the association between the risk for schizophrenia and month of birth was significant only for male early onset cases. However, as can be seen in Fig. 4, the cohorts of higher risks did not include only the cohort of November but also that of January, March, June and February. Whether this reflected the distribution of the underlying monthly related agents or mere chance fluctuation warrants further investigation.

One interpretation of our results is that individuals who are not genetically predisposed may have an increased risk of early onset schizophrenia if they were exposed to some seasonally (or monthly) varying environmental factors during the prenatal or perinatal period. Men are more susceptible to such factors than women. This is supported by evidence from many other lines of research as briefly summarized in the following.

One line of research investigated structural brain abnormalities among schizophrenic patients. Using computerized tomography to measure various parts of the brain, Zipursky and Schulz (1987) and Degreef et al. (1988) reported that patients born in the winter or early spring had more structural abnormalities than those born in other seasons. However, Owen et al. (1989) reported an opposite finding. When patients were subgrouped by family history, as reviewed by Lewis et al. (1987), most of the studies showed that patients with no family history exhibit more structural brain abnormalities than patients with a family history.

Furthermore, many studies have indicated that male or early onset schizophrenic patients are more likely to have structural brain abnormalities as well as a history of obstetric complications than their female or late onset counterparts. In a review of 10 imaging studies, Castle and Murray (1991) found that male schizophrenic patients were more likely to have structural brain abnormalities than female patients. Schizophrenic patients with early onset were found to have correlation between decreased brain area and deficits in cognitive function, while patients with late onset did not show such a relationship (Johnstone et al., 1989). Meanwhile, males with schizophrenia have earlier onset of symptoms than females with schizophrenia (Angermeyer and Kühn, 1988; Faraone et al., 1994), and males with schizophrenia are more likely than their female counterparts to have a history of obstetric complications (Pearlson et al., 1989; Foerster et al., 1991).

Another line of research showed that male schizophrenic patients are under less genetic influence than female schizophrenic patients. For example, the first-degree relatives of male schizophrenics were found to be at smaller risk of schizophrenia than the relatives of female schizophrenics (Bellodi et al., 1986; Goldstein et al., 1990; Pulver et al., 1992). Similarly, Kringlen (1987) reported that male monozygotic twins had a lower concordance rate than their female counterparts.

Integration of all these relevant findings, although controversies still exist in some of them, leads to the suggestion that a larger proportion of male, early onset schizophrenic patients would have been due to early environmental insults than 
female or late onset patients. This is consistent with our findings that the association between the risk of schizophrenia and month of birth was more prominent in non-familial, male, and early onset cases.

The nature of the environmental factor(s) that might be responsible for the season of birth effect in schizophrenia remains to be elucidated. Hare (1988) summarized the possible explanations of the season-of-birth effect into 3 major categories, i.e., parental factors (such as social class and parental age), protective factors, and constitutional damage (toxin, dietary deficiency, complications of pregnancy or birth, and infections). Among them, infectious agents have been intensively investigated (Mednick, 1988; O'Callaghan et al., 1991b; O'Callaghan et al., 1994). Recently, a common factor for schizophrenia and stillbirth (Torrey et al., 1993) or seasonal ovopathy (Pallast et al., 1994) was proposed.

In conclusion, our results replicated the previous finding that there is an association between the risk of schizophrenia and month of birth, through life table analysis. Furthermore, we found that the association was more prominent in non-familial, male and early onset cases. This supports the view that certain seasonally varying factors may increase the risk of schizophrenia, especially for those without a family history. Men are more vulnerable than women to such factors, and the schizophrenics resulting from the insult tend to have an early onset.

\section{Acknowledgment}

This work was supported by a grant from the National Institute of Health, Taiwan, ROC (DOH83-HR-306), and the Department of Health, Taiwan, ROC (DOH-77-18). The authors would like to thank Dr. Chien-Jen Chen for suggestions on an earlier version of the manuscript.

\section{References}

Angermeyer, M.C. and Kühn, L. (1988) Gender differences in age at onset of schizophrenia: an overview. Eur. Arch. Psychiatry Neurol. Sci. 237, 351-364.
Baron, M. and Gruen, R.S. (1988) Risk factors in schizophrenia: Season of birth and family history. Br. J. Psychiatry $152,460-465$.

Bellodi, L., Bussoleni, C., Scorza-Smeraldi, R., Grassi, G., Zacchetti, L. and Smeraldi, E. (1986) Family study of schizophrenia: exploratory analysis for relevant factors. Schizophr Bull. 12, 120-128.

Boyd, J.H., Pulver, A.E. and Stewart, W. (1986) Season of birth: schizophrenia and bipolar disorder. Schizophr. Bull. $12,173-186$

Bradbury, T.N. and Miller, G.A. (1985) Season of birth in schizophrenia: a review of evidence, methodology and etiology. Psychol. Bull. 98, 569-594.

Castle, D.J. and Murray, R.M. (1991) The neurodevelopmental basis of sex differences in schizophrenia. Psychol. Med. 21, 565-575.

Dalen, P. (1990) Does age incidence explain all season-of-birth effects in the literature? Schizophr. Bull. 16, 11-12.

Degreef, G., Mukherjee, S., Bilder, R. et al. (1988) Season of birth and $C T$ scan findings in schizophrenic patients. Biol. Psychiatry 24, 461-464.

Faraone, S.V., Chen, W.J., Goldstein, J.M. et al. (1994) Gender differences in the age at onset of schizophrenia: fact or artifact? Br. J. Psychiatry 164, 625-629.

Foerster, A., Lewis, S.W., Owen, M.J. et al. (1991) Pre-morbid adjustment and personality in psychosis: effects of sex and diagnosis. Br. J. Psychiatry 158, 171-176.

Goldstein, J.M. and Link, B.G. (1988) Gender and the expression of schizophrenia. J. Psychiatr. Res. 22, 141-155.

Goldstein, J.M., Faraone, S.V., Chen, W.J. et al. (1990) Sex differences in the familial transmission of schizophrenia. Br. J. Psychiatry 156, 819-826.

Hare, E. (1988) Temporal factors and trends, including birth seasonality and the viral hypothesis. In: H.A. Nasralla (Series Ed.), Handbook of Schizophrenia, Vol. 3, M.T. Tsuang and J.C. Simpson (Eds.), Nosology, Epidemiology and Genetics of Schizophrenia. Elsevier, Amsterdam, pp. 345-377.

Hwu, H.-G., Yeh, E.-K. and Chang, L.-Y. (1989) Prevalence of psychiatric disorders in Taiwan defined by the Chinese Diagnostic Interview Schedule. Acta Psychiatr. Scand. 79, 136-147.

Hwu, H.-G., Rin, H., Chen, C.-Z., Chang, S.-H., Huang, M.-G. and Yeh, L.-L. (1995) A study on the personal-familial and clinical data of psychiatric inpatients in Taiwan. Chinese Psychiatry 9, 16-31.

Johnstone, E.C., Owens, D.G.C., Bydder, G.M. et al. (1989) The spectrum of structural brain changes in schizophrenia: age of onset as a predictor of cognitive and clinical impairments and their cerebral correlates. Psychol. Med. 19, 91-103.

Kendler, K.S. (1988) The sporadic v. familial classification given aetiological heterogeneity: II. Power analyses. Psychol. Med. 18, 991-999.

Kim, C.-E., Lee, Y.-S., Lim, Y.-H. et al. (1994) Month of birth and schizophrenia in Korea: sex, family history and handedness. Br. J. Psychiatry 164, 829-831.

Kinney, D.K. and Jacobsen, B. (1978) Environmental factors 
in schizophrenia: new adoption study evidence. In: L.C. Wynne, R.L. Cromwell and S. Matthysse (Eds.), The Nature of Schizophrenia. Wiley, New York, pp. 38-51.

Kringlen, E. (1987) Contributions of genetic studies on schizophrenia. In: H. Häfner, W.F. Gattaz and W. Janzarik (Eds.), Search for the Causes of Schizophrenia. Springer-Verlag, Berlin, pp. 123-142.

Lee, E.L. (1980) Statistical Methods for Survival Data Analysis. Lifetime Learning, Belmont, CA, pp. 88-95.

Lewis, M.S. and Griffin, P.A. (1981) An explanation for the season of birth effect in schizophrenia and certain other diseases. Psychol. Bull. 89, 589-596.

Lewis, S.W., Reveley, A.M., Reveley, M.A. et al. (1987) The familial/sporadic distinction as a strategy in schizophrenia research. Br. J. Psychiatry 151, 306-313.

Lin, T.-Y. (1953) A study of the incidence of mental disorder in Chinese and other cultures. Psychiatry 16, 315-335.

Lin, T.-Y., Chu, H.M., Rin, H. et al. (1989) Effects of social change on mental disorders in Taiwan: observations based on a 15-year follow-up survey of general populations in three communities. Acta Psychiatr. Scand. 79 (Suppl. 348), 11-34.

Lo, C.W. (1985) Season of birth of schizophrenics in Hong Kong. Br. J. Psychiatry $147,212-213$.

McNeil, T.F. (1987) Perinatal influences in the development of schizophrenia. In: H. Helmchen and F.A. Henn (Eds.), Biological Perspectives in Schizophrenia. Wiley, New York, pp. 125-138.

Mednick, S.A. (1988) Adult schizophrenia following prenatal exposure to an influenza epidemic. Arch. Gen. Psychiatry 1988, 189-192.

O'Callaghan, E., Gibson, T., Colohan, H.A. et al. (1991a) Season of birth in schizophrenia: evidence for confinement of and excess of winter births to patients without a family history of mental disorder. Br. J. Psychiatry 158, 764-769.

O'Callaghan, E., Sham, P., Takei, N. et al. (1991b) Schizophrenia after prenatal exposure to 1957 A2 influenza epidemic. Lancet 337, 1248-1250.

O'Callaghan, E., Sham, P.C., Takei, N. et al. (1994) The relationship of schizophrenic births to 16 infectious diseases. $\mathrm{Br}$. J. Psychiatry 165, 353-356.

Owen, M.J., Jones, P., Lewis, S.W. et al. (1989) The relationship between season of birth and biological variables in schizophrenia. Schizophr. Res. 2, 17.

Pallast, E.G.M., Jongbloet, P.H., Streatman, H.M. et al. (1994) Excess seasonality of births among patients with schizophrenia and seasonal ovopathy. Schizophr. Bull. 20, 269-277.
Parker, G. and Balza, B. (1977) Season of birth and schizophrenia - an equatorial study. Acta Psychiatr. Scand. 56, 143-146.

Pearlson, G.D., Kim, W.S., Kubos, K.L. et al. (1989) Ventricle-brain ratio, computed tomographic density and brain area in 50 schizophrenics. Arch. Gen. Psychiatry 46, 690-697.

Peto, R., Pike, M.C., Armitage, P. et al. (1977) Design and analysis of randomized clinical trials requiring prolonged observation of each patient. II. Analysis and examples. Br. J. Cancer 35, 1-39.

Pulver, A.E., Sawyer, J.W. and Childs, B. (1981) The association between season of birth and the risk for schizophrenia. Am. J. Epidemiol. 114, 735-749.

Pulver, A.E., Stewart, W., Carpenter, J.W.T. et al. (1983) Risk factors in schizophrenia: season of birth in Maryland, USA. Br. J. Psychiatry 143, 389-396.

Pulver, A., Moorman, C.C., Brown, C.H. et al. (1990) Ageincidence artifacts do not account for the season-of-birth effect in schizophrenia. Schizophr. Bull. 16, 13-15.

Pulver, A.E., Liang, K.Y., Brown, C.H. et al. (1992) Risk factors in schizophrenia: season of birth, gender and familial risk. Br. J. Psychiatry $160,65-71$.

Sacchetti, E., Vita, A., Giobbio, G.M. et al. (1989) Risk factors in schizophrenia. Br. J. Psychiatry 155, 266-267.

Shur, E. (1982) Season of birth in high and low genetic risk schizophrenics. Br. J. Psychiatry 140, 410-415.

Shur, E. and Hare, E. (1983) Age-prevalence and the season of birth effect in schizophrenia: a response to Lewis and Griffin. Psychol. Bull. 93, 373-377.

Torrey, E.F. and Bowler, A.E. (1990) The seasonality of schizophrenic births: a reply to Mac S. Lewis. Schizophr. Bull. $16,1-3$.

Torrey, E.F. and Torrey, B.B. (1979) A shifting seasonality of schizophrenic births. Br. J. Psychiatry 134, 183-186.

Torrey, E.F., Bowler, A.E., Rawlings, R. et al. (1993) Seasonality of schizophrenia and stillbirths. Schizophr. Bull. $19,557-562$.

Watson, C.G. (1990) Schizophrenic birth seasonality and the age-incidence artifact. Schizophr. Bull. 16, 5-10.

Watson, C.G., Kucala, T., Angulski, G. et al. (1982) Season of birth and schizophrenia: a response to the Lewis and Griffin critique. J. Abnorm. Psychol. 91, 120-125.

World Health Organization (1978) Mental Disorders: Glossary and Guide to their Classification in Accordance with the Ninth Revision of the International Classification of Diseases (ICD-9) Geneva, WHO.

Zipursky, R.B. and Schulz, S.C. (1987) Seasonality of birth and CT findings in schizophrenia. Biol. Psychiatry 22, 1288-1292. 